\title{
Morphological Changes of Parotid Gland in Experimental Hyperlipidemia
}

\author{
Ioanna D. Daskala ${ }^{1}$ and Christina C. Tesseromatis ${ }^{2}$ \\ ${ }^{1}$ Faculty of Dentistry, University of Athens, 11527 Goudi, Greece \\ ${ }^{2}$ Medicine in Pharmacology Department, Faculty of Medicine, University of Athens, 11527 Goudi, Greece
}

Correspondence should be addressed to Ioanna D. Daskala, iodaska@yahoo.com

Received 23 June 2010; Revised 29 October 2010; Accepted 13 January 2011

Academic Editor: Jukka H. Meurman

Copyright ( 2011 I. D. Daskala and C. C. Tesseromatis. This is an open access article distributed under the Creative Commons Attribution License, which permits unrestricted use, distribution, and reproduction in any medium, provided the original work is properly cited.

\begin{abstract}
Objective. The aim of this study was to investigate the role of hyperlipidemia in the microstructure of parotid gland and its possible amelioration through statin treatment on Wistar rats. Methods. Forty Wistar rats $(111.06 \pm 3.36 \mathrm{~g})$ were divided into 4 groups (A1, A2 controls, B1, B2 experimental). Groups A1 and A2 consumed normal cereal rodents diet during the experimental hyperlipidemic mixture. The A2 and B2 were treated with simvastatin (Zocor) $40 \mathrm{mg} / \mathrm{kg} /$ daily p.o. for 3 months. Results. Total cholesterol, triglycerides, high density lipoproteins, and low density lipoproteins were increased in groups B1 and B2 while the parotid weight was decreased. The histological findings demonstrated changes in the parotid gland morphology of the B1 and B2, such as the presence of chronic inflammation, fibrosis, lipocytes, and foci of lymphocytic infiltration. Conclusions. The influence of statin tended to predominate over the chronic inflammation while the lipocytes were decreased and remodelling of the parotid's structure occurred.
\end{abstract}

\section{Introduction}

Hyperlipidemia is characterized by elevated levels of plasma total cholesterol (TC), triglycerides (TG), and low-density lipoprotein cholesterol (LDLC) $[1,2]$. Patients with hyperlipidemia are considered to be at high risk to develop lipids disorders that are associated with immune system deficiency, promoting manifestations including fatty degeneration of parotid parenchyma and parotid duct enlargement [3-6]. Various diseases could be associated with parotid gland swelling, such as primary or secondary Sjogren Syndrome (SS), diabetes mellitus, Wegener's granulomatosis, sialadenitis, and infections [7-9].

Recent studies have revealed that patients with SS and treated for hyperlipidemia may present a resolution of parotid symptoms [10]. Few clinical studies demonstrated that children and adolescents with hypercholesterolemia present endothelial disfunction $[11,12]$. Simvastatin reducing TC and LDL-C concentrations restores endothelial function during a short period of time $[11,12]$. Statin benefits are derived from reductions in atherogenic lipoprotein levels and increases in high-density lipoprotein cholesterol (HDL-C), $[13,14]$.

Abnormal fat deposition in the major salivary glands such as parotid gland could be detected by using shortinversion-time inversion recovery (STIR) and fat-saturation MR sequences and CT values. All three in vivo techniques confirm premature deposition of fat in the major salivary glands while the severity of fat deposition could be correlated with impaired rates of salivary flow in these patients [15-17]. MR imaging findings of salivary glands in patients with hyperlipidemia include enlargement of parotid gland, lipid infiltration, and impaired salivary flow, whereas sialography of parotid gland revealed normal findings [15-17]. Monitoring of fat deposition might be a very useful weapon in diagnosis any parotid gland's disease [15-18].

The aim of this study was to investigate the role of hyperlipidemia in the parotid gland's microstructure of Wistar rats and the influence of simvastatin treatment. 


\section{Materials and Methods}

Forty female, specific-pathogen-free Wistar rats aged 8-10 weeks (mean body weight $111.06 \pm 3.36 \mathrm{~g}$ ) were used and divided into the following four groups:

A1 control: normal cereal rodents $\operatorname{diet}(n=10)$,

A2 control: normal cereal rodents $\operatorname{diet}(n=10)$,

B1 experimental: special diet $(n=10)$,

B2 experimental: special diet $(n=10)$.

Groups B1 and B2 were treated with high fat diet in order to investigate its influence in rats (group B1). Normal cereal rodents' diet contained $100 \%$ cereals while hyperlipidemic diet consisted of $50 \%$ cereals, $20 \%$ butter, $10.5 \%$ sucrose, $10 \%$ casein, $2.5 \%$ cholesterol, $1 \%$ vitamins, $0.15 \%$ propylthiouracil and $0.1 \%$ choline [19]. Moreover, groups A2 and B2 received simvastatin (Zocor was gratefully offered by Vianex) $40 \mathrm{mg} / \mathrm{kg} /$ daily peros for 3 months via gastroesophageal cathether.

The animal experiment was performed in accordance with the institutional and national guidelines for the care and the use of experimental animals and, therefore, in compliance with the European Communities Council Directive of 24 November 1986 [20]. All animals were sacrificed under general anaesthesia and blood samples from carotid vessel were collected in order to examine the serum lipids such as TC, HDL, LDL, and TG. Parotid gland was isolated, removed, and examined. The purpose was to notify any difference in the organs' macro and microstructure. The parotid glands were preserved in $10 \%$ buffered formaldehyde fixative solution (Merck) for further histological examination. Sections of parotids' specimens were stained with haematoxylinoesin, and a full histological investigation was carried out.

Furthermore, the morphometrics features were evaluated using a microscope $\mathrm{Bx} 42$ Olympus, a camera Altra 20 Olympus, and image's analysis with Image - Pro Plus. Moreover, based on the morphometric findings, a statistical analysis was performed using Excel-Student $t$-test. Biochemical features were statistically analyzed by performing SPSS KruskalWallis Test and Mann-Whitney Test.

\section{Results}

3.1. Laboratory Findings. The hyperlipidemic diet in group B1 leads to a statistically significant increase of TC, HDL, HDL, and TG $(P<.001)$ and to an insignificant increase of parotid gland weight compared to group A1 (normal cereal diet) and A2 (normal cereal diet + simvastatin), (Table 1).

Furthermore, the simvastatin treatment in group B2 (hyperlipidemic diet + simvastatin) compared to group B1 decreased the serum levels of TC, HDL, LDL, TG and parotid gland in a statistically significant way $(P<.001)$, (Table 1$)$.

No changes were observed in group A2 compared to group A1 concerning the serum lipids profile and the parotid gland weight (Table 1). Group A2 presented a statistically insignificant decrease of parotid gland weight compared to group B1 and group B2 (Table 1). Group B2 in comparison to group $\mathrm{A} 2$ the serum levels of TC, HDL, LDL, TG, and

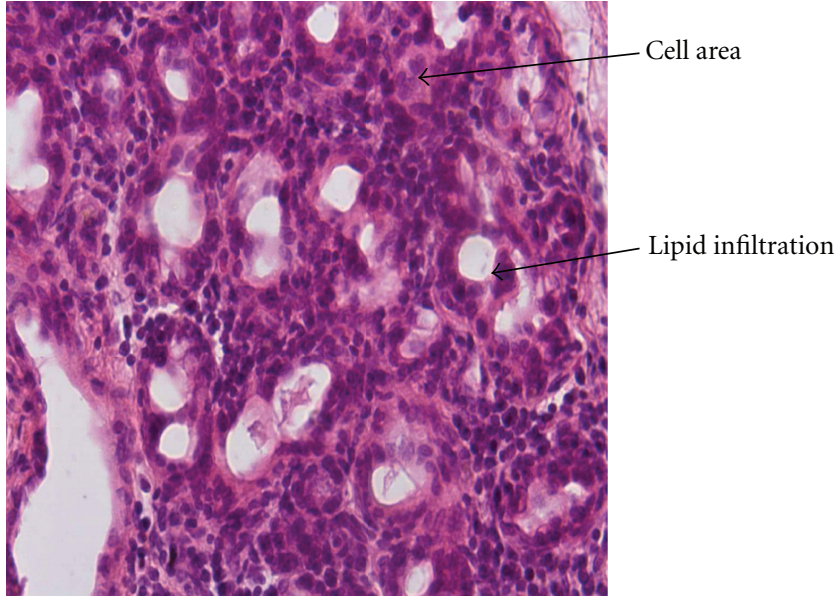

Figure 1: In group B1, lipid infiltration of the parotid parechyma, presence of lipocytes and nuclei abnormalities (increased cell area and duct's diameter, decreased duct's number) were observed (haematoxylin eosin- original magnification $\times 400$ ).

liver weight were increased in a statistically significant way $(P<.001)$, (Table 1$)$.

Finally, as far as the atheromatic index in group B1 and group B2 was concered, it should be underlined that there was a statistically insignificant difference (Table 1).

3.2. Histological Findings via Morphometry. According to histological findings, different state of lipid infiltration of the parotid gland specimens, stained with haematoxylin-oesin, in hyperlipidemic animals in group B1, B2 was observed. In addition, chronic inflammation, mild fibrosis, lypocytes, foci of lymphocytic infiltration nuclei abnormalities and diversity of vessel wall thickness were recorded $[4,18]$.

Moreover, based on the morphometrical analysis of the histological features, our research resulted in the following discoveries:

(a) Animals of group B1 compared to animals of group A1, demonstrated a statistically significant increase of cell area and duct's diameter while the duct's number was decreased $(P<.001$, Figure 1, Table 2$)$.

(b) Animals of group B2 compared to animals of group Al, revealed statistically insignificant alterations in the cell area, the number and diameter of the ducts $(P>.01$, Figure 2, Table 2).

3.3. Discussion. The goal of this research was to evaluate the microstructural alterations of parotid gland in experimental hyperlipidemia and the possible amelioration through statin treatment in Wistar rats. According to the biochemical data hyperlipidemic diet leads to an elevated serum lipid profile that might be responsible for parotid gland swelling as it is referred from Goldman and Julian, Kaltreider and Talal, Manganelli et al., and Tretbar [3, 4, 6, 19]. In addition, Izumi et al. suggest that parotid gland is more affected by hyperlipidemia than submandibular gland $[16,17]$. 
TABLE 1: Mean and standard deviation of mean of total cholosterol (TC), triglycerides (TG), high density lipoproteins (HDL), low -density lipoproteins (LDL), parotid gland-liver-thymos-spleen-adrenal weight, and atheromatic index in the four groups (A1, A2, B1, B2).

\begin{tabular}{lccrc}
\hline Groups & A1 & A2 & B1 & B2 \\
\hline TC mg/dL & $54.71 \pm 0.000^{*}$ & $65.25 \pm 0.000^{*}$ & $391.14 \pm 0.000^{*}$ & $346.25 \pm 0.000^{*}$ \\
HDL mg/dL & $17.09 \pm 0.000^{*}$ & $20.12 \pm 0.000^{*}$ & $40.86 \pm 0.000^{*}$ & $36.37 \pm 0.000^{*}$ \\
LDL mg/dL & $25.29 \pm 0.000^{*}$ & $27.25 \pm 0.000^{*}$ & $324.86 \pm 0.000^{*}$ & $285.25 \pm 0.000^{*}$ \\
TG mg/dL & $62.29 \pm 0.000^{*}$ & $89.12 \pm 0.000^{*}$ & $128.00 \pm 0.000^{*}$ & $123.00 \pm 0.000^{*}$ \\
Parotid weight mg & $37.80 \pm 2.208^{* * *}$ & $39.57 \pm 6.607^{* * *}$ & $43.71 \pm 2.556^{* * *}$ & $35.84 \pm 3.568^{* * *}$ \\
Atheromatic index & $3.20 \pm 0.98^{* * *}$ & $3.24 \pm 1.01^{* * *}$ & $9.57 \pm 0.34^{* * *}$ & $9.52 \pm 0.33^{* * *}$ \\
\hline
\end{tabular}

${ }^{*} P<.001, * * P<.01, * * * P>.01$.

TC: $\mathrm{A} 1 / \mathrm{A} 2 * * *, A 1 / B 1^{*}, \mathrm{~A} 2 / \mathrm{B} 1 *, \mathrm{~A} 2 / \mathrm{B} 2^{*}, \mathrm{~B} 1 / \mathrm{B} 2^{*}, \mathrm{~A} 1 / \mathrm{B} 2^{*}$.

HDL: A1/A2***, $\mathrm{A} 1 / \mathrm{B} 1 *, \mathrm{~A} 2 / \mathrm{B} 1 *, \mathrm{~A} 2 / \mathrm{B} 2 *, \mathrm{~B} 1 / \mathrm{B} 2 *, \mathrm{~A} 1 / \mathrm{B} 2 *$.

LDL: $\mathrm{A} 1 / \mathrm{A} 2 * * *, \mathrm{~A} 1 / \mathrm{B} 1 *, \mathrm{~A} 2 / \mathrm{B} 1^{*}, \mathrm{~A} 2 / \mathrm{B} 2 *, \mathrm{~B} 1 / \mathrm{B} 2 *, \mathrm{~A} 1 / \mathrm{B} 2^{*}$.

TG: $\mathrm{A} 1 / \mathrm{A} 2^{* * *}, \mathrm{~A} 1 / \mathrm{B} 1^{*}, \mathrm{~A} 2 / \mathrm{B} 1 *, \mathrm{~A} 2 / \mathrm{B} 2 *, \mathrm{~B} 1 / \mathrm{B} 2 *, \mathrm{~A} 1 / \mathrm{B} 2 *$.

Parotid weight: $\mathrm{A} 1 / \mathrm{A} 2 * * *, \mathrm{~A} 1 / \mathrm{B} 1 * * *, \mathrm{~A} 2 / \mathrm{B} 1^{* * *}, \mathrm{~A} 2 / \mathrm{B} 2 * * *, \mathrm{~B} 1 / \mathrm{B} 2 * * *, \mathrm{~A} 1 / \mathrm{B} 2 * * *$.

Atheromatic index: $\mathrm{A} 1 / \mathrm{A} 2 * * *, \mathrm{~A} 1 / \mathrm{B} 1^{* * *}, \mathrm{~A} 2 / \mathrm{B}^{* * *}, \mathrm{~A} 2 / \mathrm{B} 2^{* * *}, \mathrm{~B} 1 / \mathrm{B} 2^{* * *}, \mathrm{~A} 1 / \mathrm{B} 2^{* * *}$.

TABLE 2: Item Measurement parameters (statistical average value) for cells and ducts in the four groups of animals.

\begin{tabular}{|c|c|c|c|c|c|}
\hline & Density (ave) & Density (std) & Diameter & Ratios (PER area \%) & Aspect ratio \\
\hline \multicolumn{6}{|l|}{ Cells } \\
\hline Group A1 & 74.621 & 7.248 & 5.589 & $7.154 \%$ & 1.481 \\
\hline Group A2 & 71.890 & 6.969 & 5.663 & $9.409 \%$ & 1.472 \\
\hline Group B1 & 76.413 & 7.722 & 6.658 & $5.660 \%$ & 1.531 \\
\hline Group B2 & 78.066 & 7.950 & 5.877 & $12.361 \%$ & 1.546 \\
\hline \multicolumn{6}{|l|}{ Ducts } \\
\hline Group A1 & 130.849 & 35.091 & 33.736 & $1.545 \%$ & 1.281 \\
\hline Group A2 & 131.985 & 37.292 & 34.437 & $1.481 \%$ & 1.301 \\
\hline Group B1 & 135.055 & 36.146 & 41.140 & $0.633 \%$ & 1.218 \\
\hline Group B2 & 135.064 & 36.740 & 40.838 & $2.388 \%$ & 1.369 \\
\hline
\end{tabular}

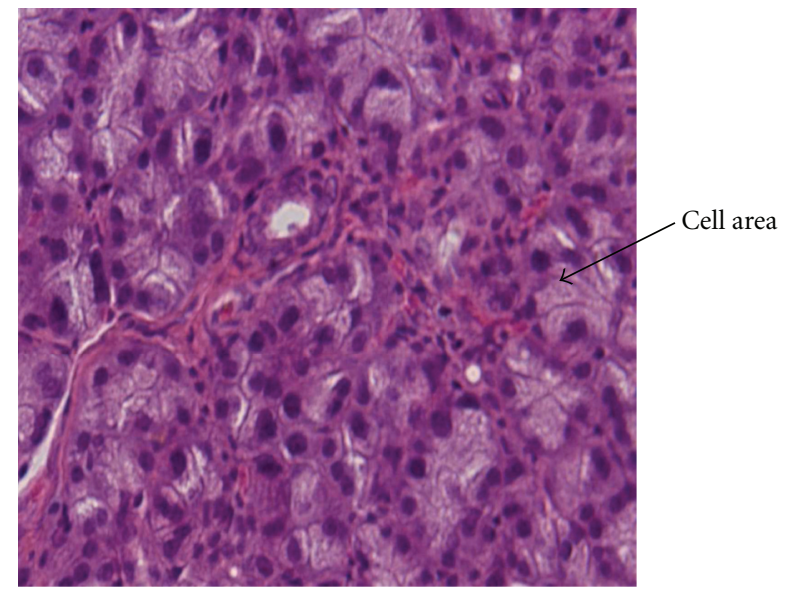

FIGURE 2: In group B2, there were not significant alterations in the cell area or the number and the diameter of the ducts of the parotid gland (haematoxylin eosin-original magnification $\times 400$ ).

The above results are in accordance with the findings of Sheikh et al., suggesting a close relationship between parotid gland swelling and high levels of triglycerides in plasma $[10,16,17]$.
Moreover, it is not well known how hyperlipidemia causes parotid gland swelling. Larsson et al. used the Folch method in order to extract the lipids from parotid, submandibular,and whole stimulated saliva in human [21]. According to Folch et al., in this method, the tissue is homogenized with Chloroform/methanol (2/1) to a final volume 20 times the volume of the tissue sample and after dispersion the whole mixture is agitated during 15-20 minutes in a orbital shaker at room temperature. The homogenate is either filtrated or centrifuged to recover the liquid phase. The solvent is washed with 0.2 volumes $(4 \mathrm{~mL}$ for $20 \mathrm{~mL}$ ) of water or better $0.9 \% \mathrm{NaCl}$ solution. After vortexing some seconds, the mixture is centrifuged at low speed to separate the two phases. After centrifugation and siphoning of the upper phase, the lower chloroform phase containing lipids is evaporated under vacuum in a rotary evaporator or under a nitrogen stream if the volume is under 2-3 mL [22]. Larsson's findings indicate that saliva lipids mainly consist of cholesterol esters, cholesterol, triglycerides, diglycerides, monoglycerides, and free fatty acids [21]. According to Hindy et al., the saliva gland parenchyma is considered to be a place of sterols biosynthesis, similar to those of liver, as it was proven by using thin-layer and gas-liquid chromatography [23].

Man et al. demonstrated changes of the pancreas morphology by hypertriglyceridemia. It seems that triglycerides 
(TGs) are stored in pancreatic islets, which inhibited glucoseinduced insulin secretion, in part, via reduced glucokinase activity in the islets [24]. Several studies have suggested that pancreas and parotid gland may present, in part, morphological and functional similarities [25-28]. Therefore, Anderson et al. studied salivary tissues of diabetic male Wistar rats in order to distinguish the differences in intracellular lipids between diabetic and controls glands. Lipid accumulation within parenchymal cells depended on the type of the gland and their content of serous cells. Parotid and sublingual glands possess the greatest amount of lipids compared to submandibular gland. Their findings may explain the mild alteration of the submandibular gland in patients with hyperlipidemia contrary to the parotid gland's involvement [29].

Moreover, this report reveals that the hyperlipidemic diet may result to no statistically significant decrease of parotid weight, observed in the hyperlipidemic-experimental group compared to controls. It is of great interest that hyperlipidemia, except for lipid deposition and lymphocytic infiltration of parotid gland, causes alterations of the parenchyma as well.

Furthermore, simvastatin treatment resulted in a statistically significant decrease of serum lipid profile but to a statistically insignificant decrease of parotid gland weight. Statins seem to repair parotid gland's changes caused by hyperlipidemia, resulting eventually to gland remodelling [11].

Due to our findings, a close relationship between high plasma lipid levels and parotid gland enlargement may be concluded. This enlargement might be an indication of parotid gland's microstructural changes, and it may explain itxs observed, by other investigators, functional impairment. It is obvious that statins, reducing the serum lipid profile, may play a protective role in organs' injuries caused by hyperlipidemia by repairing the occurred alterations.

\section{References}

[1] R. M. Krauss, "Triglycerides and atherogenic lipoproteins: rationale for lipid management," American Journal of Medicine, vol. 105, no. 1A, supplement 1, 1998.

[2] S. M. Grundy, "Hypertriglyceridemia, atherogenic dyslipidemia, and the metabolic syndrome," American Journal of Cardiology, vol. 81, no. 4A, pp. 18-25, 1998.

[3] J. A. Goldman and E. H. Julian, "Pseudo Sjogren syndrome with hyperlipoproteinemia," Journal of the American Medical Association, vol. 237, no. 15, pp. 1582-1584, 1977.

[4] H. B. Kaltreider and N. Talal, "Bilateral parotid gland enlargement and hyperlipoproteinemia," Journal of the American Medical Association, vol. 210, no. 11, pp. 2067-2070, 1969.

[5] R. Abaurre and J. Vega, "Enlargement of the parotid glands and its relation to metabolic and cardiovascular changes," Medicina, vol. 40, no. 2, pp. 161-166, 1980.

[6] P. Manganelli, F. Salaffi, and U. Ambanelli, “Pseudo-Sjogren's syndrome in type IV hyperlipoproteinemia. Description of a clinical case," Minerva Medica, vol. 80, no. 9, pp. 1031-1033, 1989.

[7] B. E. Chegar and R. T. Kelley, "Wegener's granulomatosis presenting as unilateral parotid enlargement," Laryngoscope, vol. 114, no. 10, pp. 1730-1733, 2004.
[8] R. M. Gislon da Silva, "Captopril-induced bilateral parotid and submandibular sialadenitis," European Journal of Clinical Pharmacology, vol. 60, no. 6, pp. 449-453, 2004.

[9] C. Mourouzis, T. Saranteas, M. Tsamouris, and C. Tesseromatis, "Morphological changes of parotid glands following adjuvant arthritis and ibuprofen treatment in rats," International Journal of Oral and Maxillofacial Surgery, vol. 32, no. 1, pp. 69-73, 2003.

[10] J. S. Sheikh, M. Sharma, A. Kunath, D. A. Fritz, C. J. Glueck, and E. V. Hess, "Reversible parotid enlargement and pseudosjögren's syndrome secondary to hypertriglyceridemia," Journal of Rheumatology, vol. 23, no. 7, pp. 1288-1291, 1996.

[11] W. P. Ferreira, M. C. Bertolami, S. N. Santos et al., "Onemonth therapy with simvastatin restores endothelial function in hypercholesterolemic children and adolescents," Pediatric Cardiology, vol. 28, no. 1, pp. 8-13, 2007.

[12] M. C. Ling, T. D. Ruddy, R. A. Dekemp et al., "Early effects of statin therapy on endothelial function and microvascular reactivity in patients with coronary artery disease," American Heart Journal, vol. 149, no. 6, article e9, p. 1137, 2005.

[13] S. E. Nissen, E. M. Tuzcu, P. Schoenhagen et al., "Reversal of atherosclerosis with aggressive lipid lowering (REVERSAL) investigators. Statin therapy, LDL cholesterol, C-reactive protein, and coronary artery disease," The New England Journal of Medicine, vol. 352, no. 1, pp. 29-38, 2005.

[14] S. J. Nicholls, E. M. Tuzcu, I. Sipahi et al., "Statins, highdensity lipoprotein cholesterol, and regression of coronary atherosclerosis," Journal of the American Medical Association, vol. 297, no. 5, pp. 499-508, 2007.

[15] M. Izumi, K. Eguchi, H. Nakamura, S. Nagataki, and T. Nakamura, "Premature fat deposition in the salivary glands associated with Sjogren syndrome: MR and CT evidence," American Journal of Neuroradiology, vol. 18, no. 5, pp. 951958, 1997.

[16] M. Izumi, A. Hida, Y. Takagi, Y. Kawabe, K. Eguchi, and T. Nakamura, "MR imaging of the salivary glands in sicca syndrome: comparison of lipid profiles and imaging in patients with hyperlipidemia and patients with Sjoegren syndrome," American Journal of Roentgenology, vol. 175, no. 3, pp. 829834, 2000.

[17] P. Manganelli, F. Salaffi, and U. Ambanelli, "Pseudo-Sjogren's syndrome in type IV hyperlipoproteinemia. Description of a clinical case," Minerva Medica, vol. 80, no. 9, pp. 1013-1033, 1989.

[18] J. Pijpe, W. W. I. Kalk, J. E. van der Wal et al., "Parotid gland biopsy compared with labial biopsy in the diagnosis of patients with primary Sjögren's syndrome," Rheumatology Advance Access, vol. 371, 2006.

[19] H. C. Tretbar, "Parotid enlargement and hyperlipidemia," Arizona Medicine, vol. 28, no. 4, pp. 261-262, 1971.

[20] "European Communities Directive of 1986 regarding the protection of animals used for experimental and other scientific purposes(86/609/EEC)," http://www.europa.eu/enviroment/protection of nature and biodiversity/protection of animals used for experimental purposes.

[21] B. Larsson, G. Olivecrona, and T. Ericson, "Lipids in human saliva," Archives of Oral Biology, vol. 41, no. 1, pp. 105-110, 1996.

[22] J. Folch, M. Lees, and G. H. Sloane Stanley, "A simple method for the isolation and purification of total lipides from animal tissues," The Journal of Biological Chemistry, vol. 226, no. 1, pp. 497-509, 1957. 
[23] A. M. Hindy, K. Yoshiga, K. Takada, and K. Okuda, "Sterol composition and biosynthesis in mouse salivary glands," Archives of Oral Biology, vol. 31, no. 2, pp. 87-93, 1986.

[24] Z. W. Man, M. Zhu, K. Toide et al., "Impaired beta-cell function and deposition of fat droplets in the pancreas as a consequence of hypertriglyceridemia in OLETF rat, amodel of spontaneous NIDDM," Diabetes, vol. 46, no. 11, pp. 17181724, 1997.

[25] J. Axelson, B. Fan, B. Ohlsson, J. Rehfeld, M. Ekelund, and I. Ihse, "The changes in the rat parotid glands following total parenteral nutrition and pancreatico-biliary diversion are not mediated by cholecystokinin," International Journal of Gastrointestinal Cancer, vol. 2, pp. 109-111, 1996.

[26] J. H. Won, W. J. Cottrell, T. H. Foster, and D. I. Yule, "Ca2 release dynamics in parotid and pancreatic exocrine acinar cells evoked by spatially limited flash photolysis," American Journal of Physiology, vol. 293, no. 6, pp. G1166-G1177, 2007.

[27] V. Kreuder, J. Dieckhoff, M. Sittig, and H. G. Mannherz, "Isolation, characterisation and crystallization of deoxyribonuclease I from bovine and rat parotid gland and its interaction with rabbit skeletal muscle actin," European Journal of Biochemistry, vol. 139, no. 2, pp. 389-400, 1984.

[28] R. J. Charchaflie, L. Bustos Fernandez, C. J. Perec, E. Gonzalez, and A. Marzi, "Functional studies of the parotid and pancreas glands in amyotrophic lateral sclerosis," Journal of Neurology Neurosurgery and Psychiatry, vol. 37, no. 7, pp. 863-867, 1974.

[29] L. C. Anderson and J. R. Garrett, "Lipid accumulation in the major salivary glands of streptozotocin-diabetic rats," Archives of Oral Biology, vol. 31, no. 7, pp. 469-475, 1986. 


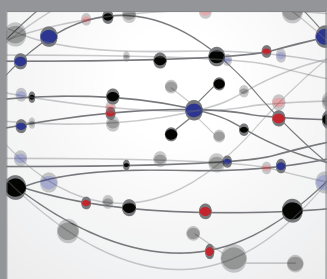

The Scientific World Journal
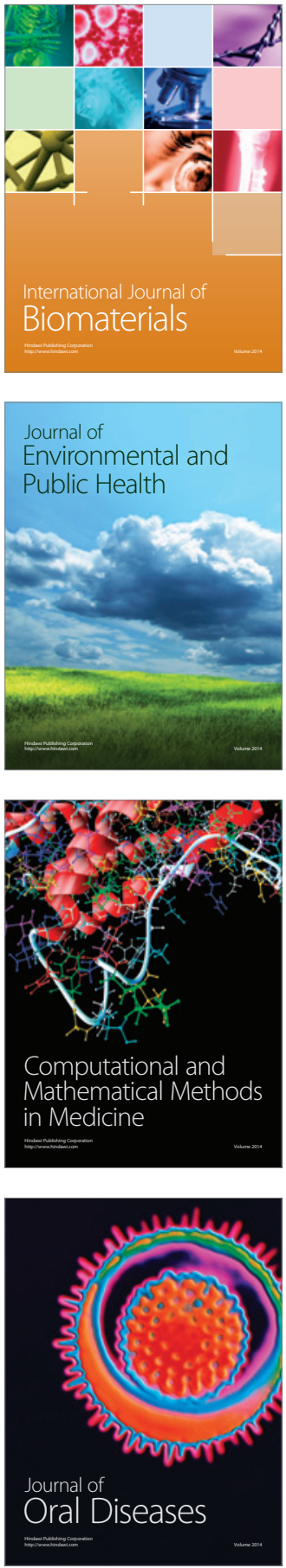
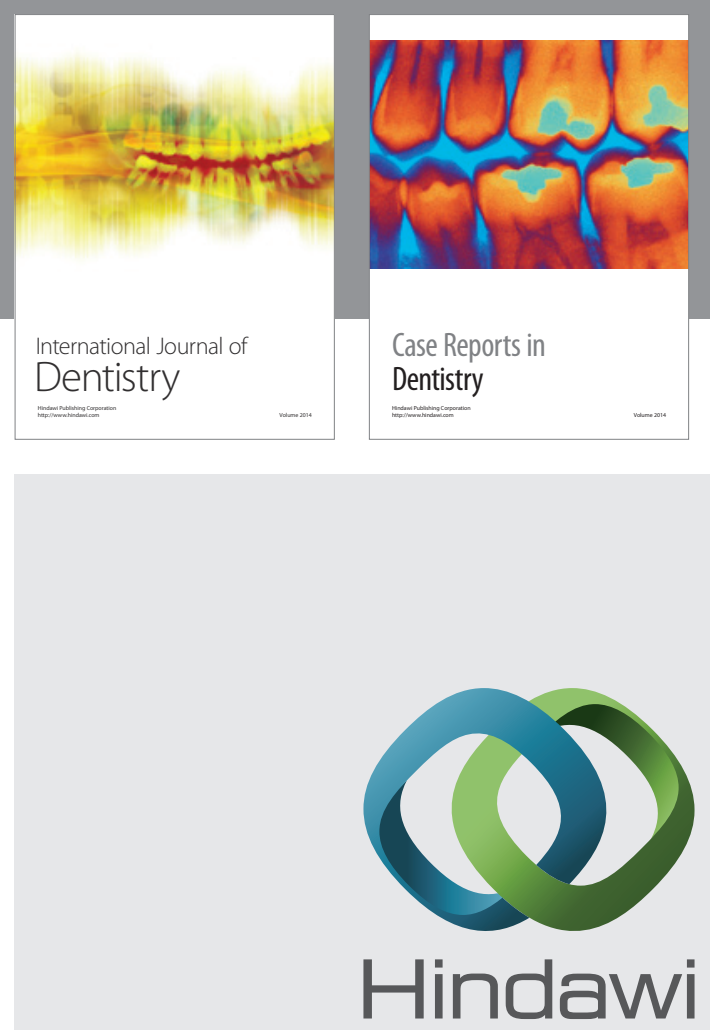

Submit your manuscripts at

http://www.hindawi.com
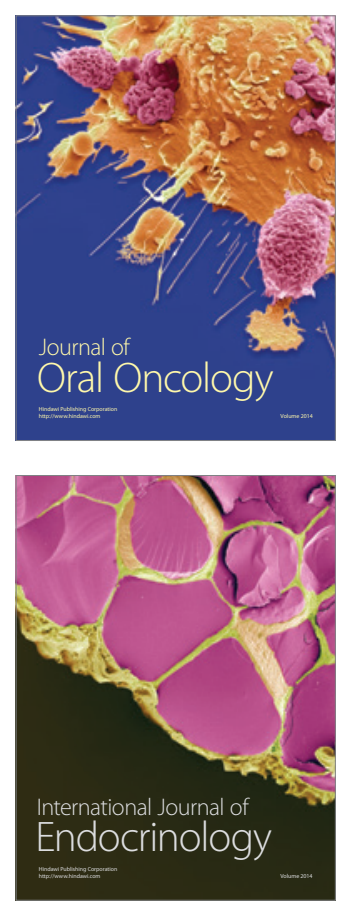
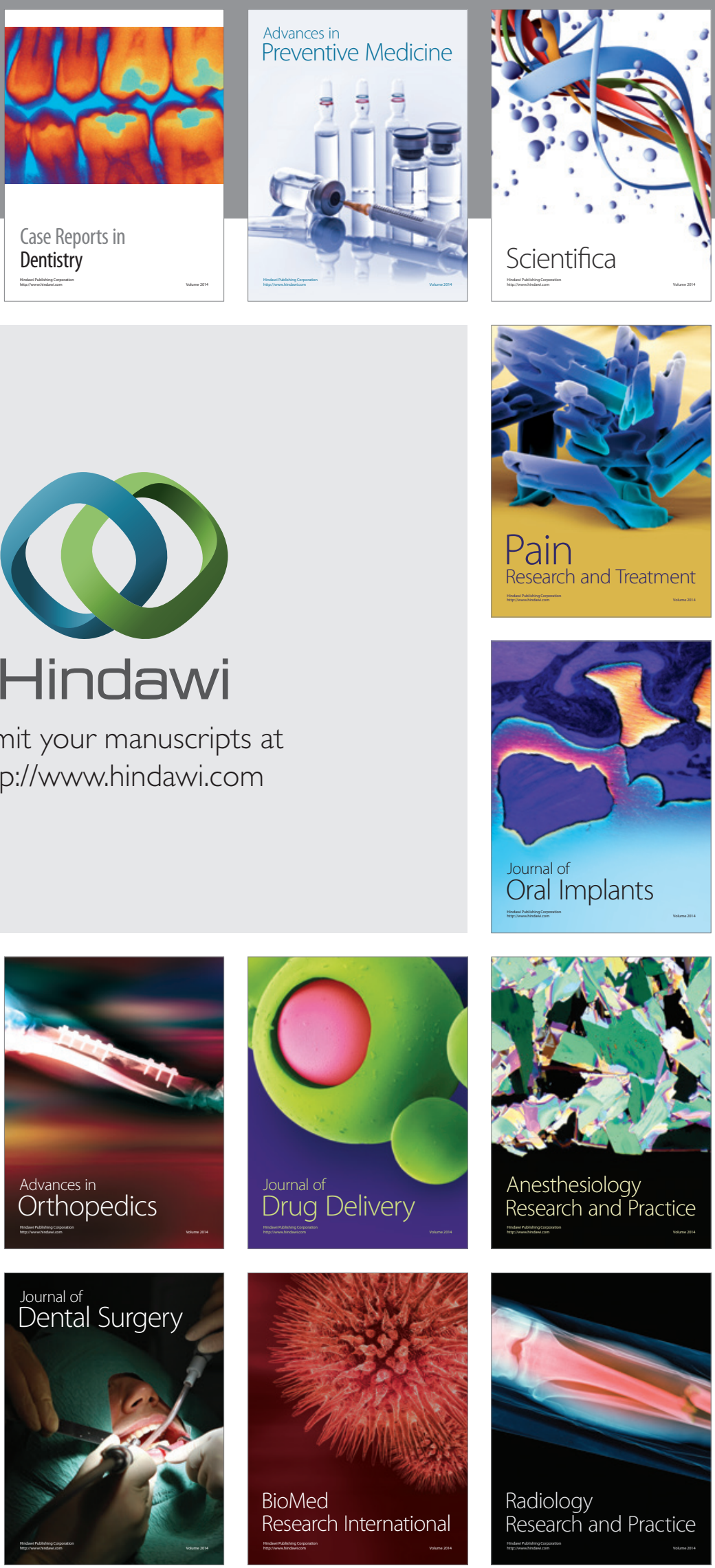